\title{
ARTICLE OPEN \\ Clinical whole genome sequencing as a first-tier test at a resource-limited dysmorphology clinic in Mexico
}

Alicia Scocchia ${ }^{1}$, Kristen M. Wigby ${ }^{2,3}$, Diane Masser-Frye ${ }^{2}$, Miguel Del Campo ${ }^{2,3}$, Carolina I. Galarreta ${ }^{2,3}$, Erin Thorpe ${ }^{1}$, Julia McEachern $^{1}$, Keisha Robinson ${ }^{1}$, Andrew Gross ${ }^{1}$, ICSL Interpretation and Reporting Team, Subramanian S. Ajay ${ }^{1}$, Vani Rajan ${ }^{1}$, Denise L. Perry ${ }^{1}$, John W. Belmont ${ }^{1}$, David R. Bentley ${ }^{1}$, Marilyn C. Jones (iD ${ }^{2,3}$ and Ryan J. Taft ${ }^{1}$

Patients with rare, undiagnosed, or genetic disease (RUGD) often undergo years of serial testing, commonly referred to as the "diagnostic odyssey". Patients in resource-limited areas face even greater challenges-a definitive diagnosis may never be reached due to difficulties in gaining access to clinicians, appropriate specialists, and diagnostic testing. Here, we report on a collaboration of the Illumina iHope Program with the Foundation for the Children of the Californias and Hospital Infantil de Las Californias, to enable deployment of clinical whole genome sequencing (cWGS) as first-tier test in a resource-limited dysmorphology clinic in northern Mexico. A total of 60 probands who were followed for a suspected genetic diagnosis and clinically unresolved after expert examination were tested with cWGS, and the ordering clinicians completed a semi-structured survey to investigate change in clinical management resulting from cWGS findings. Clinically significant genomic findings were identified in $68.3 \%(n=41)$ of probands. No recurrent molecular diagnoses were observed. Copy number variants or gross chromosomal abnormalities accounted for $48.8 \%(n=20)$ of the diagnosed cases, including a mosaic trisomy and suspected derivative chromosomes. A qualitative assessment of clinical management revealed $48.8 \%(n=20)$ of those diagnosed had a change in clinical course based on their cWGS results, despite resource limitations. These data suggest that a cWGS first-tier testing approach can benefit patients with suspected genetic disorders.

npj Genomic Medicine (2019)4:5 ; https://doi.org/10.1038/s41525-018-0076-1

\section{INTRODUCTION}

There are estimated to be more than 200 million people worldwide with an unresolved rare genetic disease. ${ }^{1}$ An additional $\sim 4 \%$ of births worldwide per year will be affected by a genetic disorder. ${ }^{2}$ Genetic disease is tied to substantial healthcare cost and lost productivity burdens, ${ }^{3}$ although the relative impact is expected to be higher in resource-limited geographies with disadvantaged access to care. A historical analysis of global hemoglobin disorder screening indicates that genetic disorders only become a priority once a country achieves an infant mortality rate of less than 40 per 1000 live births, ${ }^{4}$ suggesting that as the mortality associated with communicable diseases, poor nutrition, and lack of resources declines the relative burden of genetic diseases increases. In resource-rich geographies, pediatric patients with a genetic disorder can account for more than a third of hospital admissions and more than $50 \%$ of total charges. ${ }^{5}$ The only systematic survey of genetic disease in a large Mexican hospital showed similar results: more than a third of admissions were associated with a genetic disease, and these patients had more frequent and longer hospital stays and an increased number of surgeries. ${ }^{5,6}$

Clinical genetic assessment of patients with suspected genetic disorders is fundamental to subsequent treatment, but interrogating across clinically heterogeneous phenotypes often involves a process of serial testing for specific conditions. This strategy can be expensive and time-consuming, fostering the possibility of incomplete testing and failure to achieve a molecular diagnosis in many patients. ${ }^{7}$ Clinical whole genome sequencing (cWGS) holds promise as a singular testing platform that allows for the simultaneous interrogation of known disease genes and detection of single nucleotide variants (SNVs), small insertions and/or deletions (indels), copy number variants (CNVs), and some structural chromosomal anomalies. ${ }^{7-10}$ Evidence is accumulating to support the use of cWGS as a first-tier test for patients with suspected Mendelian or chromosomal disease where diagnosis is not possible from clinical examination alone. .,10,11 $^{-1}$

Here, we report on a cohort receiving cWGS through a partnership between Illumina's iHope Program and the Foundation for the Children of the Californias (US-based 501c3) which supports Hospital Infantil de Las Californias, a non-profit, resourcelimited pediatric facility in Baja California, Mexico. For many patients seen at this facility, access to multiple specialists and serial molecular testing to obtain diagnoses is typically not logistically or financially feasible. These underserved pediatric patients represent a unique population in which the effectiveness of cWGS as a first-tier test can be assessed.

\footnotetext{
'Illumina, Inc, San Diego, CA 92122, USA; ${ }^{2}$ Rady's Children's Hospital, San Diego, CA 92123, USA and ${ }^{3}$ University of California, San Diego, CA 92093, USA Correspondence: Marilyn C. Jones (mjones@rchsd.org) or Ryan J. Taft (rtaft@illumina.com)

These authors contributed equally: Alicia Scocchia and Kristen M. Wigby

These authors jointly supervised this work: Marilyn C. Jones and Ryan J Taft
}

Received: 27 August 2018 Accepted: 19 December 2018

Published online: 14 February 2019 


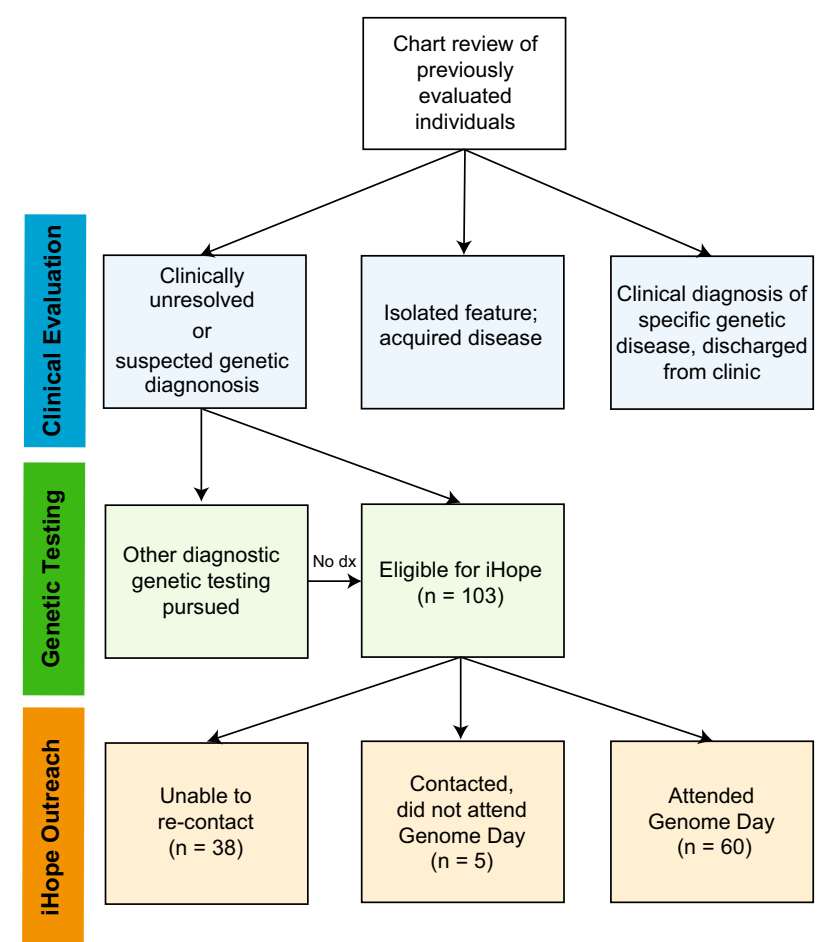

Fig. 1 Case selection criteria. Chart review of previously evaluated individuals was performed by the clinician team. Probands who were diagnosed with a recognizable pattern of malformation (e.g., isolated Down syndrome), received counseling, and discharged from clinic were excluded from referral to the iHope Program. Probands with acquired disease (e.g.,: suspected environmental exposures) or isolated features (e.g.,: individuals with cleft lip with or without cleft palate) were typically also excluded. Probands with prior non-diagnostic molecular or cytogenic testing were included if all other criteria were met. Resulting families who were eligible for the iHope Program were contacted, offered cWGS, and scheduled to attend a Genome Day. Upon completion of a Genome Day visit, whole-blood samples were transported to the clinical laboratory for cWGS. Dx: diagnosis; cWGS: clinical whole genome sequencing

\section{RESULTS}

Of 103 families deemed eligible for the iHope Program after chart review, sixty probands $(58.3 \%)$ were successfully contacted and attended a "Genome Day", a clinic visit dedicated to cWGS testing consent and phenotyping. Of those individuals who did not participate, the majority was due to inability to re-contact the family to offer cWGS ( $n=38 ; 79 \%)$. The remaining five families were successfully contacted and initially expressed interest in cWGS but did not follow-up with a visit to clinic for a Genome Day (Fig. 1). Six Genome Days were held between August 2016 and March 2018 in which 60 probands and their families (one proband-only, 14 duos, 42 trios, and three quads) provided informed consent and blood samples for cWGS (Table 1). No families who attended a Genome Day and participated in the informed consent process declined to participate in CWGS testing. The mean rounded proband age was 7.6 years at the time of blood draw (ranging from four months to 21 years). Most $(n=41$; $68.3 \%$ ) probands had no prior cytogenetic or molecular genetic testing. Of the probands that underwent some genetic testing prior to cWGS, karyotype analysis was the most commonly completed test $(n=19)$. Three probands who underwent karyotype analysis also pursued other genetic testing, including single gene analysis $(n=1)$, methylation studies to assess for Prader-Willi syndrome $(n=1)$, and chromosomal microarray $(n=1)$. The most common indications for testing included congenital anomalies, developmental delay, seizures/epilepsy, growth restriction, and intellectual disability, with clinician categorization showing $76.7 \%$ $(46 / 60)$ of proband phenotypes were consistent with a suspected pattern of malformation and $23.3 \%(14 / 60)$ with a primary neurologic presentation.

A genomic finding congruent with the reported phenotype was identified in 41 of 60 probands, resulting in an overall diagnostic yield of $68.3 \%$ (Table 2). In 36 probands, pathogenic or likely pathogenic variants were reported in genes or regions of the genome with significant phenotypic overlap, which were considered positive molecular diagnoses. Three probands were considered to have likely positive molecular diagnoses, where variants of unknown significance (VUS) were reported in wellcharacterized genes, which were confirmed to contribute to the proband's diagnosis by subsequent clinical feedback (P6, P20, P36). Two cases were considered partial molecular diagnoses, as the identified pathogenic variant was hypothesized to explain the proband's phenotype only in part (P17 and P31). Of note, molecular diagnoses were achieved for $80 \%(12 / 15)$ of all duo and proband only cases. A diagnostic result from cWGS was obtained for $76.1 \%$ of all probands in the patterns of malformation phenotype group compared with $42.9 \%$ of all probands in the primary neurologic phenotype group $(p=0.0455)$. All individuals in this cohort received analysis for ACMG secondary findings, and pathogenic secondary findings were reported in three cases (P6, $\mathrm{P} 12$, and P39). These include one maternally inherited pathogenic $B R C A 2$ variant identified in a trio case, one de novo pathogenic deletion encompassing the entire STK11 gene in a trio case, and one pathogenic deletion involving exons 1-10 of the PMS2 gene in the proband but not in the proband's mother identified in a duo case.

A range of variant types were observed in the 41 cases in which primary molecular diagnoses were achieved. These included SNVs $(n=18 ; 43.9 \%)$, CNVs ranging from $26 \mathrm{~kb}-18 \mathrm{Mb}(n=10 ; 24.4 \%)$, multiple terminal CNVs suggestive of derivative chromosomes ( $n$ $=5 ; 12.2 \%)$, aneuploidies ( $n=2 ; 4.9 \%)$, absence of heterozygosity $(\mathrm{AOH})$ consistent with uniparental isodisomy (UPD) $(n=1 ; 2.4 \%)$, indel $(n=2 ; 4.9 \%)$, a compound heterozygous variant pair involving multiple variant types $(n=1 ; 2.4 \%)$, a dual diagnosis of SNV and aneuploidy $(n=1 ; 2.4 \%)$, and one case with at least four molecular diagnoses including a SNV and a compound heterozygous pair involving multiple variant types $(n=1 ; 2.4 \%)$, as summarized in Fig. 2.

No molecular diagnosis was achieved in 19 cases. In four negative cases, birth injury or teratogenic exposures including monozygotic twin-related injury, valproic acid embryopathy, fetal alcohol syndrome, and Zika virus-related embryopathy, were noted by the clinician team as among the differential diagnoses. In one negative case, the proband is suspected to have a genetic disorder involving mosaicism given her hyperpigmented and hypopigmented patchy skin lesions on the legs and skeletal asymmetry including hypoplasia of the left humerus and shoulder girdle. In some negative cases $(n=4 ; 6.7 \%)$, variants of interest were reported for clinical consideration (Table 3 ). These probands are not considered to have received molecular diagnoses, but clinicians have characterized these variants of clinical interest as either possibly contributory $(n=3)$ or secondary $(n=1)$ molecular findings in relation to the proband's phenotype.

Surveys assessing the clinical utility of cWGS results were completed by the clinical team for all 60 probands. Of the 41 probands who received a molecular diagnosis, cWGS results produced new clinical diagnoses in $80.5 \%$ of cases $(n=33)$ and confirmed clinical diagnoses in $19.5 \%$ of cases $(n=8)$. A change in the proband's clinical course due to cWGS findings was reported in $48.8 \%$ of cases $(n=20)$. These included referrals to specialists to assesses for co-morbidities ( $n=8$; e.g., neurology, ophthalmology, audiology) or for imaging or functional testing ( $n=6$; e.g., renal ultrasound, brainstem auditory evoked response test, echocardiogram, electrocardiogram, electroencephalogram). Muscle biopsy 


\begin{tabular}{|c|c|c|}
\hline & \multicolumn{2}{|c|}{ Cohort $(n=60)$} \\
\hline & $n$ & $\%$ \\
\hline Sex (male) & 30 & 50.0 \\
\hline \multicolumn{3}{|l|}{ Age } \\
\hline Birth -2 years & 11 & 18.3 \\
\hline $3-8$ years & 25 & 41.7 \\
\hline $9-14$ years & 20 & 33.3 \\
\hline 15-21 years & 4 & 6.7 \\
\hline \multicolumn{3}{|l|}{ Phenotype } \\
\hline Neurological presentation & 14 & 23.3 \\
\hline Pattern of malformation & 46 & 76.7 \\
\hline \multicolumn{3}{|l|}{ cWGS analysis type } \\
\hline Proband-only & 1 & 1.7 \\
\hline Duo & 14 & 23.3 \\
\hline Trio & 42 & 70.0 \\
\hline Quad & 3 & 5.0 \\
\hline \multicolumn{3}{|l|}{ Prior genetic testing } \\
\hline Karyotype & 19 & 31.7 \\
\hline$(+)$ Single gene testing & (1) & \\
\hline (+) PWS methylation studies & (1) & \\
\hline (+) Chromosomal microarray & (1) & \\
\hline None & 41 & 68.3 \\
\hline \multicolumn{3}{|l|}{ Genome day } \\
\hline 1 (Aug 2016) & 7 & 11.7 \\
\hline 2 (Nov 2016) & 10 & 16.7 \\
\hline $3(\operatorname{Jan} 2017)$ & 8 & 13.3 \\
\hline 4 (June 2017) & 8 & 13.3 \\
\hline 5 (September 2017) & 14 & 23.3 \\
\hline 6 (March 2018) & 13 & 21.7 \\
\hline
\end{tabular}

was avoided in three probands who received molecular diagnoses, reducing potential morbidity and clinical resource burden. Some findings $(n=3)$ led to additional clinical investigations related to the molecular diagnosis, including abnormal eye movements further investigated for possible seizures and examination of muscle tone in association with progressive spastic paraplegia. One family received information regarding the creation of an augmentative communication system for a child with Angelman syndrome, a condition in which there is typically poor prognosis for the development of expressive verbal language. One patient was transitioned to palliative care after molecular diagnosis of neuronal ceroid lipofuscinosis. Finally, current or future screening for malignancies and/or tumors/polyps was discussed for the proband with a deletion encompassing exons 1-10 of the PMS2 gene (P6), for the proband with an entire gene deletion of STK11 (P39), and for the proband and mother with a pathogenic $B R C A 2$ variant (P12) identified by cWGS as secondary findings.

Among cases where no molecular diagnoses were achieved, cWGS was reported to be helpful to the proband's clinical care in at least six probands and their families. In two cases where the proband's brain imaging suggested possible leukodystrophy, cWGS was reported to be helpful to clinicians in communicating a significantly reduced likelihood for suspected specific diagnoses. In three negative cases, clinicians were motivated to expedite additional clinical work-up to further investigate the proband's phenotype, including ophthalmology exams and a muscle biopsy. For one proband (P43) with no molecular diagnosis, a variant of clinical interest was submitted to GeneMatcher ${ }^{12}$ and yielded contact from three institutions and enrollment in a clinical study of patients with suspected causative variants in the USP7 gene.

Post-test genetic counseling was modified for 49 families (100\% of probands with molecular diagnoses; $81.7 \%$ of the cohort) following cWGS results. Among cases with molecular diagnoses, the most frequently cited reason for the change in post-test counseling was the ability to share information about recurrence risk/options for preconception testing or prenatal diagnosis $(n=$ $37)$ and prognostic indications $(n=21)$. Among negative cases, the most frequently cited reasons for change in post-test counseling was ability to counsel regarding decreased likelihood of genetic conditions $(n=3)$.

\section{DISCUSSION}

In this cohort summary, we have shown that deployment of cWGS as a first-tier test in a resource-limited clinic is able to identify diverse, clinically significant genomic variation in the majority (68\%) of probands with suspected genetic disease, almost half of whom had a change in clinical course due to the molecular findings. The ability to detect a wide breadth of disease-causing genomic variation via a single test offers potential benefit for a variety of patients, including those with a broad differential diagnosis, disease-causing variants of different types, and/or with dual diagnoses. For example, in an 11-year old male proband (P40), a paternally inherited SNV and a maternally inherited 46-bp deletion in the ECEL1 gene were identified simultaneously using cWGS, consistent with autosomal recessive distal arthrogryposis. A dual diagnosis was obtained in a child with clinically diagnosed atypical Down syndrome (P41), which included long myopathic facies, scapular winging, tapered calves, infantile spasms, severe muscle weakness, and hypotonia, who was found to harbor both trisomy 21 and a maternally inherited, likely pathogenic ACTA1 SNV associated with nemaline myopathy. Four molecular diagnoses were reported in a 14-year-old female with a phenotype including developmental and cognitive delays, epilepsy, growth deficiency with lack of secondary sexual characteristics, and dysmorphic features (P39), with contribution from both SNVs and a CNV. Two of these diagnoses were considered primary diagnoses, consistent with the patient's current reported phenotype, while the other two diagnoses were secondary or incidental findings. This individual may not have received all molecular diagnoses by conventional clinical serial testing strategies, even in a resource-rich area, as the likelihood of continuing the testing process is low once an initial finding is obtained.

The detection of CNVs $(n=15)$, aneuploidies $(n=2)$, and dual diagnoses involving copy number variation $(n=3)$ accounted for $48.8 \%(20 / 41)$ of diagnostic findings. This likely reflects population-specific resource limitations, including reduced access to cytogenetic testing and very limited access to chromosomal microarray testing prior to cWGS. It is possible that many of these variants would have been detected by microarray, but the cost of microarray remains prohibitively expensive for many families served in this resource-limited dysmorphology clinic. We therefore opted to utilize donated cWGS as a first-line test, as opposed to a reflex for patients with non-diagnostic microarray testing, so as not to exclude families with financial barriers to accessing microarray. As the cost of WGS continues to decline, future studies may directly assess the cost-effectiveness and clinical implications of first-tier WGS testing against reflexive WGS or exome sequencing after non-diagnostic microarray.

There is also accumulating evidence that WGS out-performs microarray in both sensitivity and specificity. For example, a prospective study of patients referred for pediatric genetics evaluation showed a four-fold increase in diagnostic yield with WGS over chromosomal microarray alone. ${ }^{10}$ These data also highlight the distinctive diagnostic benefits of WGS, including the 


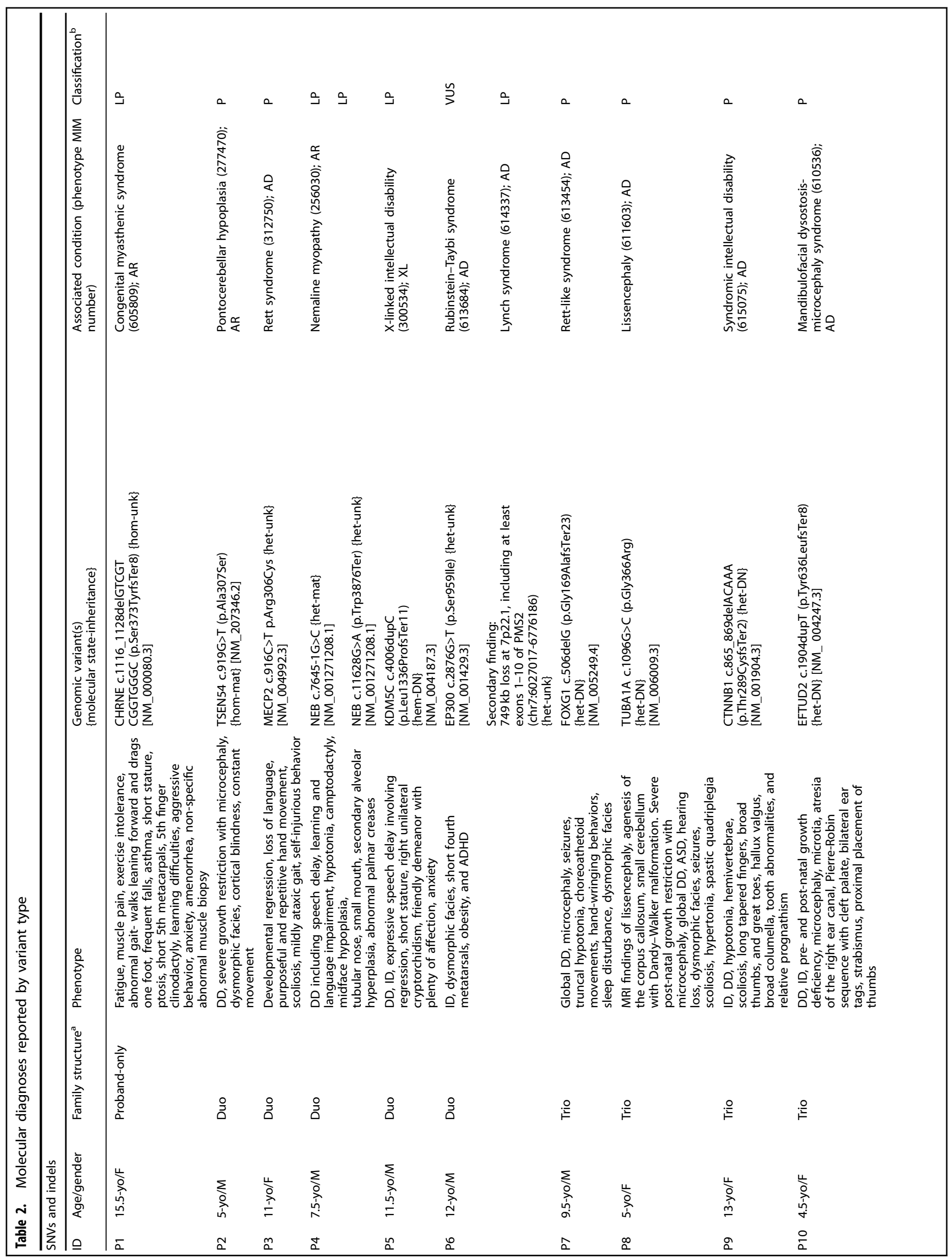




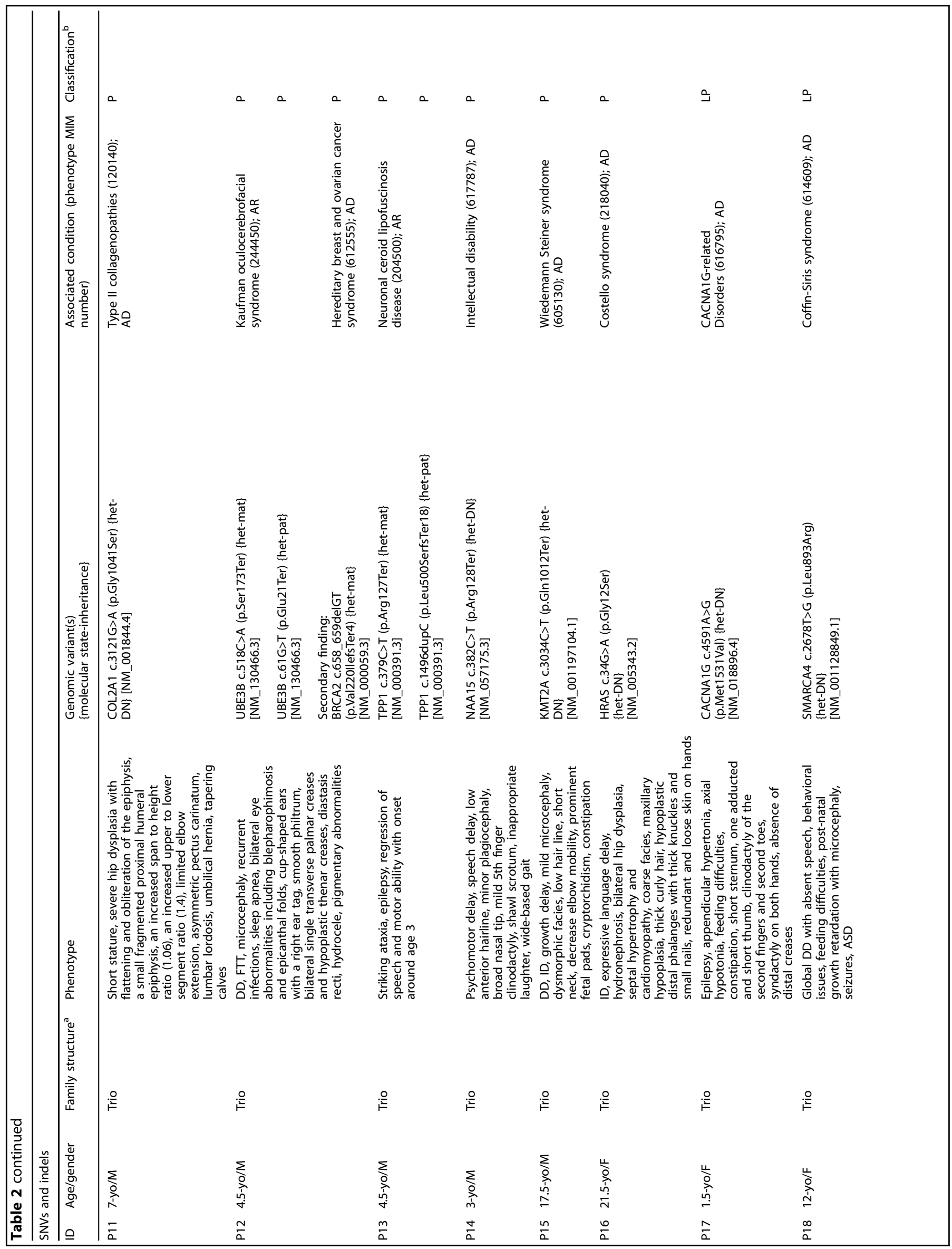




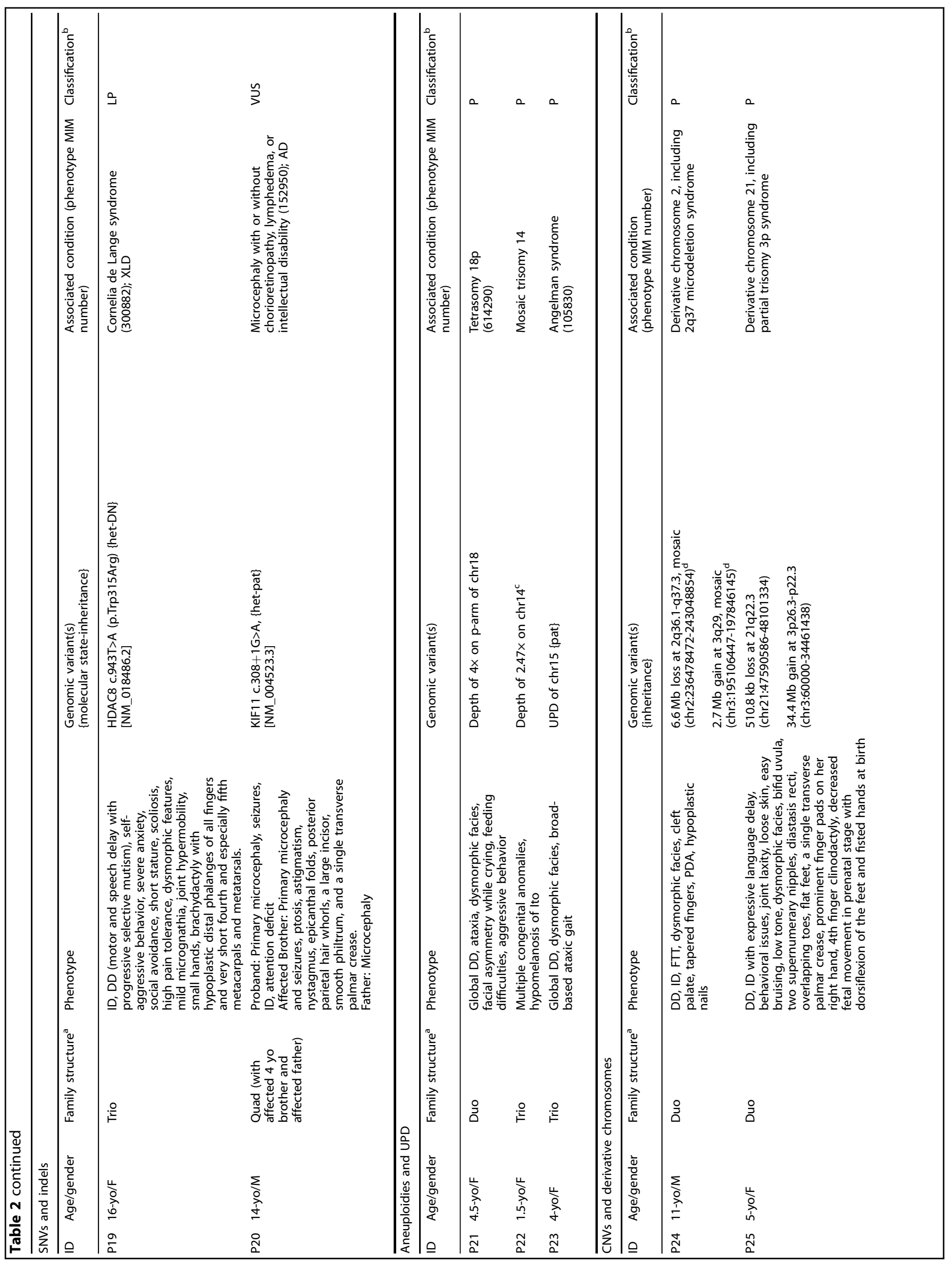




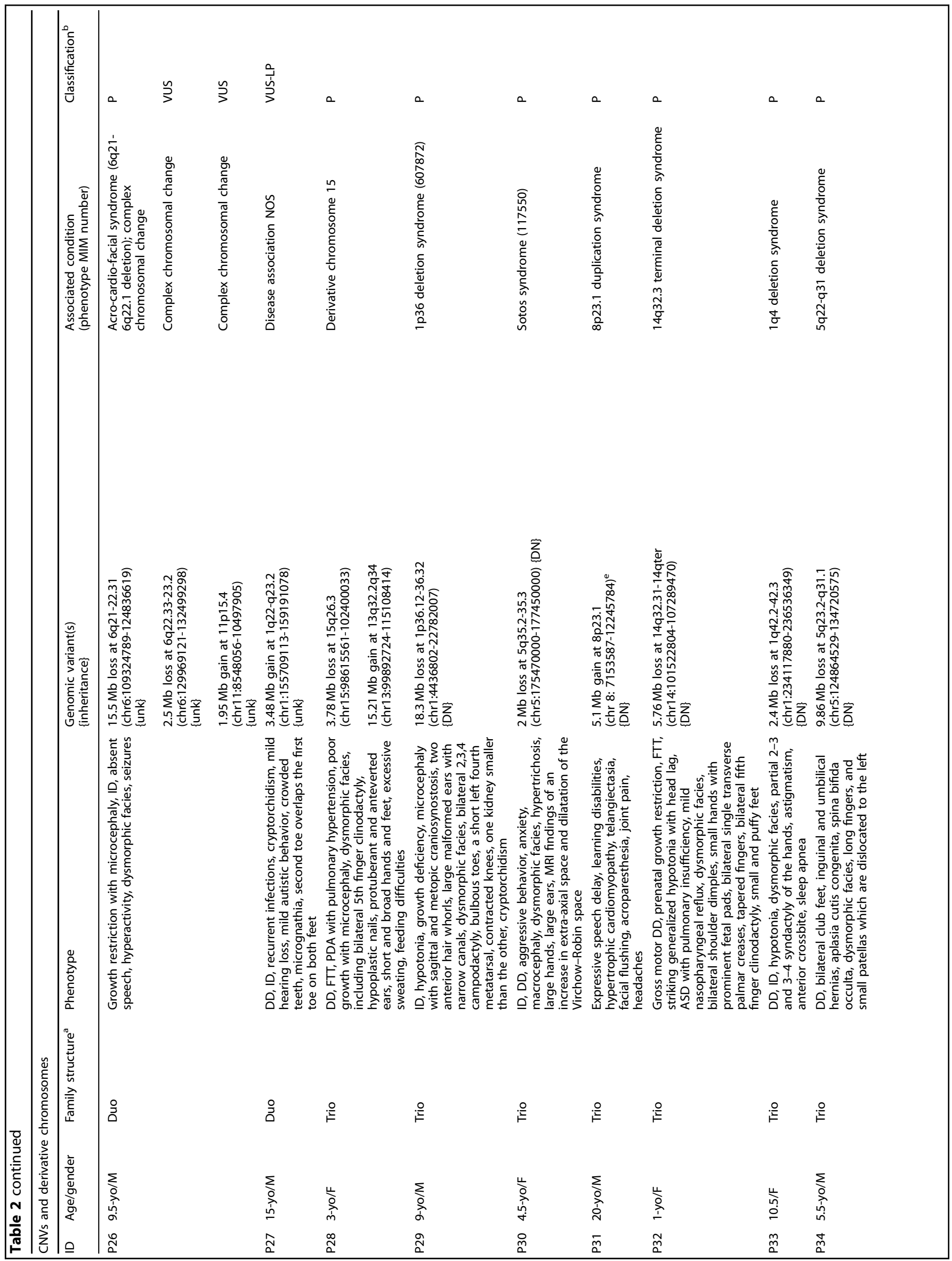




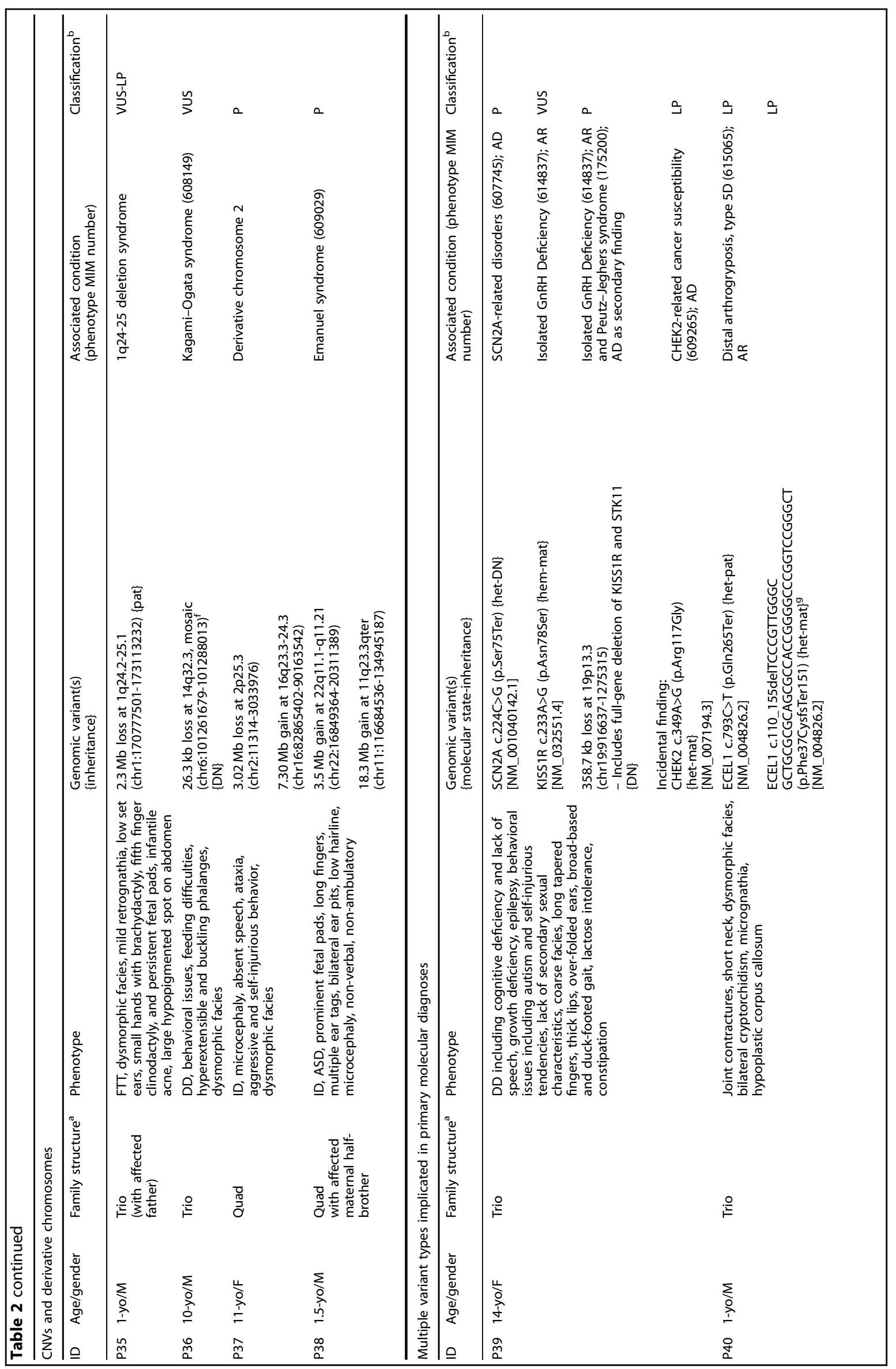




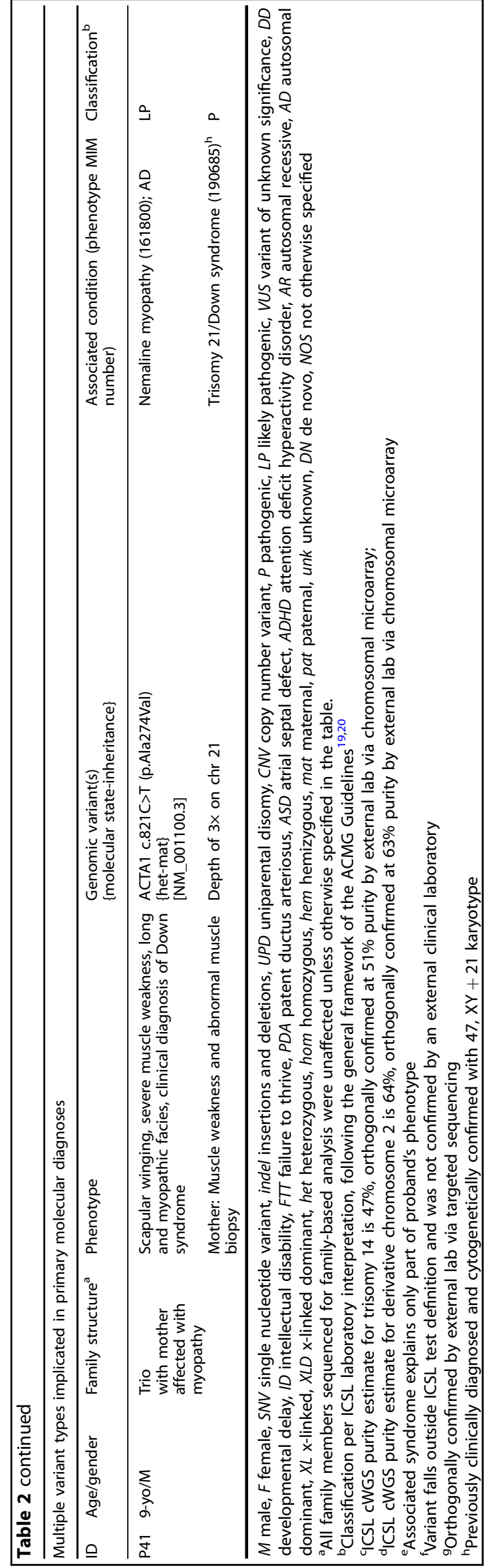

Copy number/chromosomal variants

Multiple variant types

Indels $(1 \mathrm{bp}-13 \mathrm{bp})$

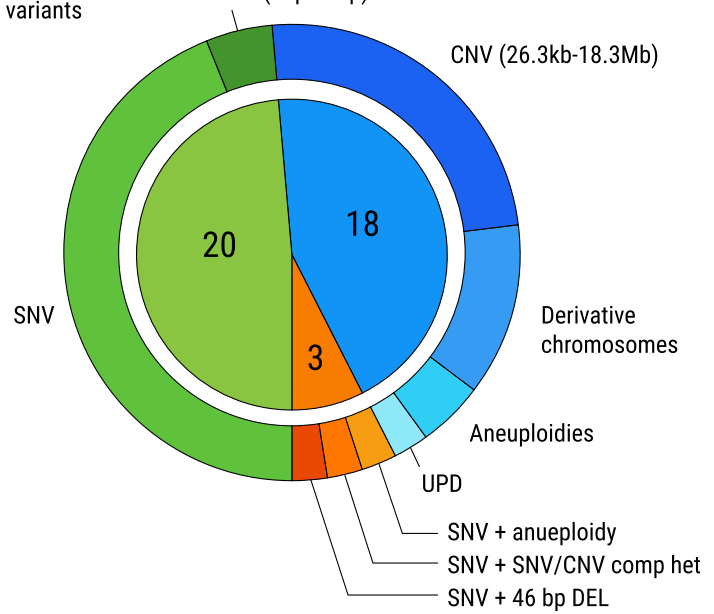

Fig. 2 Proportion of variant types observed in cases where molecular diagnoses were achieved. Number of probands with small variants (including SNVs and indels), copy number/chromosomal variants (including CNVs, derivative chromosomes, aneuploidies, and UPD), and multiple variant types (SNVs and another variant type in a single case) are noted. SNV: single nucleotide variant, indel: insertions and deletions, UPD: uniparental disomy, CNV: copy number variant

detection of single or multiple molecular diagnoses resulting from both pathogenic SNVs and CNVs in a single test. Additionally, a recent systematic evaluation of CNV calling in the context of a cWGS testing found that cWGS showed greater sensitivity for pathogenic CNVs across all size ranges. ${ }^{13}$ This cohort also provides some examples of CNVs detected by cWGS that may have been missed by other approaches, including a mosaic $26.3 \mathrm{~kb}$ deletion at $14 q 32.2$ that was detected in a 9-year-old proband (P36) with developmental delay, neonatal respiratory and feeding difficulties, and distinctive features (full cheeks, myopathic facies, anteverted nares, and hyperextensible fingers). ${ }^{13}$ This locus contains two differentially methylated regions (DMRs) involved in the imprinted regulation of MEG3. The maternal deletion of these regions has previously been associated with Kagami-Ogata syndrome ${ }^{12}$ (MIM 608149) and shows considerable phenotypic overlap with P36. Given the size and location of this finding, it may not have been detected by some clinically available microarray or exome tests.

Clinicians' survey responses endorsed the clinical utility of cWGS, with changes reported in both the proband's clinical management and post-test genetic counseling. Although a change in post-test genetic counseling was expected for most probands with molecular diagnoses, altered counseling was reported for over three quarters of the cohort, including those that did not receive a molecular diagnosis. In these cases, the negative cWGS results did not support the suspicion of genetic diagnoses and modified the provision of counseling regarding decreased likelihood of genetic conditions. In families with increased screening recommendations due to secondary findings, additional financial burden placed on the family was considered by the clinical team. Modified screening may be recommended (e.g., colon cancer screening to include CBC for anemia and fecal occult blood test initially, with follow-up colonoscopy if indicated after these initial tests) to help mitigate this burden.

Overall, these results suggest that cWGS as a first-tier test can achieve a wide range of molecular diagnoses which influence care, even in a resource-limited setting. If cWGS can be deployed at reasonable cost, this may be a helpful testing strategy in resourcelimited populations where a serial testing approach would be 


\section{METHODS}

Patient selection information

Clinical whole genome sequencing tests were philanthropically provided through Illumina's iHope Program to patients seen at the dysmorphology clinic held monthly at the Hospital Infantil de Las Californias. Case acceptance criteria for cWGS testing through the iHope Program included: (1) referral from pediatrician to the dysmorphology clinic for evaluation of congenital anomalies and/or suspected genetic disorder; (2) clinical genetics evaluation to include medical history, three generation pedigree, and physical examination with attention to major and minor malformations; and (3) inability of the family to receive appropriate genetic testing due to financial hardship. Patients were ascertained concurrently and through chart review of previously evaluated individuals (Fig. 1). Families eligible for the iHope Program were invited to participate in a "Genome Day": a visit to the dysmorphology clinic in Mexico that included an updated clinical examination, facilitation of informed consent, and blood draws of all consented family members. Informed consent for cWGS testing was facilitated in Spanish and obtained in accordance with the rules and standard operating procedures of the Illumina Clinical Services Laboratory and the Hospital Infantil de las Californias, including a review of the possibility to receive secondary findings related to highly penetrant genetic disease per recommendations from the American College of Medical Genetics and Genomics (ACMG). ${ }^{13}$ Whole-blood samples were submitted for the TruGenome ${ }^{\mathrm{TM}}$ Undiagnosed Disease cWGS test at the Illumina Clinical Services Laboratory, a CLIA-approved and CAP-accredited laboratory located at Illumina Inc in San Diego, California. Subsequent retrospective analysis of patient data from this cohort was conducted in accordance with requirements of approval by the Western Institutional Review Board.

\section{TruGenome $^{\mathrm{TM}}$ undiagnosed disease cWGS test}

The TruGenome ${ }^{T M}$ Undiagnosed Disease test is designed to detect and report on SNVs, small indels, CNVs, and mitochondrial DNA SNVs that impact genes which have an established association to genetic disease. Whole genome sequencing was performed on DNA extracted from wholeblood and prepared for next-generation sequencing using the Illumina TruSeq $^{\text {TM }}$ PCR-free kit. Samples were sequenced on the Illumina HiSeq ${ }^{\text {TM }} X^{\mathrm{TM}}$ system with paired-end 150 base pair reads at the Illumina Clinical Services Laboratory in San Diego, California, USA. The data were aligned ${ }^{14}$ according to build 37 of the Human Reference Genome (http://www. ncbi.nlm.nih.gov/projects/genome/assembly/grc/human), and analyzed using the Strelka ${ }^{15}$ caller for SNVs and Canvas ${ }^{16}$ caller for CNVs.

Genomes were sequenced to an average of $\geq 30$ fold coverage. The range of mean depth of coverage across this cohort of 167 samples was $32 \times$ to $42 x$. Interpretation was performed for SNVs and indels that fall within $15 \mathrm{bp}$ of a RefSeq exon boundary. Based on the quality filters and through the analysis of an extended, multi-generation family set (Platinum Genomes), ${ }^{17}$ sensitivity for SNVs is $98.9 \%$ and sensitivity for insertions up to 31 bases and deletions up to 27 bases is $80-85 \%$. This assay has the capability to detect copy number events greater than $10 \mathrm{~kb}$, however sensitivity was only assessed for events greater than $20 \mathrm{~kb}$ and was found to be approximately $85 \%{ }^{13}$ CNV interpretation was limited to events that either overlapped an exon or had a boundary that was within $1 \mathrm{~kb}$ upstream or downstream of an exon. Mitochondrial SNVs detected at an allele fraction greater than or equal to $3 \%$ were interpreted for 
pathogenicity for a samples analyzed during and after June 2017; however, percentage of heteroplasmy was not a component of the validated cWGS laboratory test at the time of this testing. The sensitivity metrics reported above reflect the most current test definition, and can range within 1-2\% for some samples due to test development and validation of evolving clinical laboratory assays over time. Variants of interest identified by cWGS and reported to the clinicians which were not within the clinical test definition were orthogonally tested through external clinical laboratories, if possible.

\section{Variant assessment}

Variants of interest were identified based on consideration of population allele frequency, variant consequence, evolutionary conservation, occurrence in a gene with an established gene-disease relationship, occurrence in a gene whose disease association overlaps with the patient's reported phenotype, and inheritance mode, as appropriate. A directed query of the Online Mendelian Inheritance In Man (OMIM) database was performed for each case using terms reflecting the proband's phenotype. The resultant gene list was used to prioritize variants from the family-based analysis. Variant interpretation for SNVs and CNVs was performed according to the ACMG guidelines for variant classification ${ }^{18,19}$ and a clinical report was issued for the proband to include genomic findings of potential clinical significance. Ancillary reports for secondary findings and a pharmacogenomics screen were also provided for all individuals who received cWGS.

\section{Assessment of clinical care in the context of cWGS findings}

To investigate the clinical impact of cWGS findings, semi-structured surveys designed to assess the impact of cWGS results on clinical care and post-test genetic counseling modifications were administered to the clinical team (Supplementary Table 1). The survey is comprised of multiplechoice questions as well as free-text explanations. Survey responses were utilized to report on the following: (1) if the cWGS test contributed to the proband's diagnosis; (2) if a change in clinical course was recommended or may be considered based on the cWGS results and (3) if any additional clinical actions resulting from the cWGS test occurred. A contribution to the proband's diagnosis was determined if clinicians reported that the cWGS result(s) produced a new diagnosis or confirmed a clinical diagnosis. A change in clinical course is defined as a change in management and/or reports of additional clinical testing or follow-up for the proband, which was directly related to the cWGS result. Results from multiple-choice questions were tabulated, and free-text explanations were used to provide additional details and examples.

The clinician team stratified probands into two groups based on phenotype characteristics: suspected pattern of malformation and primary neurologic presentation. Phenotypes classified as suspected patterns of malformation include those with multiple organ systems affected, dysmorphic features, and other variable clinical presentations expected to indicate a pleiotropic syndrome. The classification of neurological presentation included generally non-dysmorphic probands in whom the primary phenotype was neurological in origin. This enabled the team to determine if there were differences in molecular diagnostic success between these two broad phenotypic groups in this cohort. Statistical comparison of the diagnostic yields between different phenotype groups was performed using a Fisher's exact test.

\section{DATA AVAILABILITY}

The data that support the findings of this study are available from Illumina Clinical Services Laboratory, but restrictions apply to the availability of these data due to them containing sensitive information that could compromise patient privacy/ consent and so are not publicly available.

\section{ICSL INTERPRETATION AND REPORTING TEAM}

Maren Bennett ${ }^{1}$, Krista Bluske ${ }^{1}$, Carolyn M. Brown ${ }^{1}$, Amanda Buchanan ${ }^{1}$, Brendan Burns ${ }^{1}$, Nicole J. Burns ${ }^{1}$, Anjana Chandrasekhar ${ }^{1}$, Aditi Chawla ${ }^{1}$, Amanda R Clause ${ }^{1}$, Alison J. Coffey ${ }^{1}$, Maria Laura Cremona ${ }^{1}$, Vlad Gainullin ${ }^{1}$, R. Tanner Hagelstrom ${ }^{1}$, Alka Malhotra', Maya Rajan', Revathi Rajkumar ${ }^{1}$, Sarah Schmidt ${ }^{1}$

\section{ACKNOWLEDGEMENTS}

We are very grateful to the families in the iHope Program, the Foundation for the Children of the Californias, and the Hospital Infantil de Las Californias for their partnership in these efforts. We would like to recognize Illumina for its support of the iHope Program. Thanks to the Illumina Clinical Services Laboratory (Southern California Clinical Services) team for the sequencing and bioinformatics support, to Brittany Noble for manual review of data in tables, and to Stacie Taylor, Kirsten Curnow, and Egor Dolzhenko for formatting and figure assistance.

\section{ADDITIONAL INFORMATION}

Supplementary information accompanies the paper on the npj Genomic Medicine website (https://doi.org/10.1038/s41525-018-0076-1).

Competing interests: A.S., A.G., J.E., K.R., V.R., E.T., S.S.A., D.L.P., D.R.B., J.W.B., R.J.T., and all members of the ICSL interpretation and reporting team are employees and shareholders of Illumina Inc. The remaining authors declare no competing interests.

Publisher's note: Springer Nature remains neutral with regard to jurisdictional claims in published maps and institutional affiliations.

\section{REFERENCES}

1. Shire. Rare disease impact report: Insights from patients and the medical community. https://globalgenes.org/wp-content/uploads/2013/04/ShireReport-1.pdf (2013).

2. Verma, I. C. \& Puri, R. D. Global burden of genetic disease and the role of genetic screening. Semin. Fetal Neonatal Med. 20, 354-363 (2015).

3. Schieppati, A., Henter, J. I., Daina, E. \& Aperia, A. Why rare diseases are an important medical and social issue. Lancet 371, 2039-2041 (2008).

4. Modell, B. \& Kuliev, A. The history of community genetics: the contribution of the haemoglobin disorders. Community Genet. 1, 3-11 (1998).

5. McCandless, S. E., Brunger, J. W. \& Cassidy, S. B. The burden of genetic disease on inpatient care in a children's hospital. Am. J. Hum. Genet. 74, 121-127 (2004).

6. Carnevale, A., Hernandez, M., Reyes, R., Paz, F. \& Sosa, C. The frequency and economic burden of genetic disease in a pediatric hospital in Mexico City. Am. J. Med. Genet. 20, 665-675 (1985).

7. Lionel, A. C. et al. Improved diagnostic yield compared with targeted gene sequencing panels suggests a role for whole-genome sequencing as a first-tier genetic test. Genet. Med. https://doi.org/10.1038/gim.2017.119 (2017)

8. Trujillano, D. et al. Clinical exome sequencing: results from 2819 samples reflecting 1000 families. Eur. J. Hum. Genet. 25, 176-182 (2017).

9. Meienberg, J., Bruggmann, R., Oexle, K. \& Matyas, G. Clinical sequencing: is WGS the better WES? Hum. Genet. 135, 359-362 (2016).

10. Stavropoulos, D. J. et al. Whole-genome sequencing expands diagnostic utility and improves clinical management in pediatric medicine. NPJ Genom. Med. 1 https://doi.org/10.1038/npjgenmed.2015.12 (2016).

11. Gilissen, C. et al. Genome sequencing identifies major causes of severe intellectual disability. Nature 511, 344-347 (2014)

12. Sobreira, N., Schiettecatte, F., Valle, D. \& Hamosh, A. GeneMatcher: a matching tool for connecting investigators with an interest in the same gene. Hum. Mutat. 36, 928-930 (2015).

13. Gross, A. et al. Copy number variants in clinical WGS: deployment and interpretation for rare and undiagnosed disease. bioRxiv. https://doi.org/10.1101/ 245100 (2018).

14. Raczy, C. et al. Isaac: ultra-fast whole-genome secondary analysis on Illumina sequencing platforms. Bioinformatics 29, 2041-2043 (2013).

15. Saunders, C. T. et al. Strelka: accurate somatic small-variant calling from sequenced tumor-normal sample pairs. Bioinformatics 28, 1811-1817 (2012)

16. Roller, E., Ivakhno, S., Lee, S., Royce, T. \& Tanner, S. Canvas: versatile and scalable detection of copy number variants. Bioinformatics 32, 2375-2377 (2016).

17. Eberle, M. A. et al. A reference data set of 5.4 million phased human variants validated by genetic inheritance from sequencing a three-generation 17-member pedigree. Genome Res. 27, 157-164 (2017).

18. Kalia, S. S. et al. Recommendations for reporting of secondary findings in clinical exome and genome sequencing, 2016 update (ACMG SFv2.0): a policy statement of the American College of Medical Genetics and Genomics. Genet. Med. 19, 249-255 (2017)

19. Kearney, H. M., Thorland, E. C., Brown, K. K., Quintero-Rivera, F. \& South, S. T. American College of Medical Genetics standards and guidelines for interpretation and reporting of postnatal constitutional copy number variants. Genet. Med. 13, 680-685 (2011).

20. Richards, S. et al. Standards and guidelines for the interpretation of sequence variants: a joint consensus recommendation of the American College of Medical 
Genetics and Genomics and the Association for Molecular Pathology. Genet. Med. 17, 405-424 (2015).

(c) (i) Open Access This article is licensed under a Creative Commons Attribution 4.0 International License, which permits use, sharing, adaptation, distribution and reproduction in any medium or format, as long as you give appropriate credit to the original author(s) and the source, provide a link to the Creative Commons license, and indicate if changes were made. The images or other third party material in this article are included in the article's Creative Commons license, unless indicated otherwise in a credit line to the material. If material is not included in the article's Creative Commons license and your intended use is not permitted by statutory regulation or exceeds the permitted use, you will need to obtain permission directly from the copyright holder. To view a copy of this license, visit http://creativecommons. org/licenses/by/4.0/.

(c) The Author(s) 2019 